\title{
Aspergillus quadrilineatus revealed to be fungicolous on Phlebopus spongiosus from northern Thailand
}

\section{Gajanayake $A J^{1,2}$, Jayawardena $\mathbf{R S}^{1,2^{*}}$, Bundhun $\mathbf{D}^{1,2}$, Thongklang $\mathrm{N}^{1,2}$, Luangharn $\mathbf{T}^{\mathbf{1}}$}

Center of Excellence in Fungal Research, Mae Fah Luang University, Chiang Rai 57100, Thailand.

${ }^{2}$ School of Science, Mae Fah Luang University, Chiang Rai 57100, Thailand.

Gajanayake AJ, Jayawardena RS, Bundhun D, Thongklang N, Luangharn T. 2021 - Aspergillus quadrilineatus revealed to be fungicolous on Phlebopus spongiosus from northern Thailand. Fungal Biotec 1(1), 26-41, Doi 10.5943/FunBiotec/1/1/3

\begin{abstract}
Fungicolous fungi are able to grow on both cultivable and wild mushrooms as saprotrophs, mycoparasites or symbionts. These taxa are capable of causing deformations or decomposition of mushrooms and can also be potential pathogens on cultivable mushrooms, thereby causing both yield and quality losses. Therefore, accurate identification and investigation of taxonomic diversity of the fungicolous taxa associated with mushrooms would be beneficial for disease management strategies and quarantine purposes in the mushroom industry. During a cultivation trial to grow Phlebopus spongiosus on soil, the mushrooms were contaminated with an Aspergillus species. Multi-locus phylogeny based on combined ITS, tub2, cam and rpb2 sequence data and morphological characters revealed the fungicolous contaminant to be A. quadrilineatus. Herein A. quadrilineatus is described with complete descriptions, illustration and a phylogenetic tree of Aspergillus section Nidulantes to show its placement. This study is the first report of $A$. quadrilineatus as a fungicolous species on Phlebopus spongiosus.
\end{abstract}

Keywords - Aspergillaceae - Eurotiales - Eurotiomycetes - host record - taxonomy

\section{Introduction}

Phlebopus spongiosus Pham and Har. Takah., is an ectomycorrhizal mushroom which is edible and native to southern Vietnam (Kumla et al. 2020, Raghoonundon et al. 2021). It produces medium to large, dark yellowish-brown fruiting bodies with a sponge-like tissue more or less similar to $P$. portentosus, but can be distinguished by its dark brown spore print and distinctive hymenial cystidia (Pham et al. 2012). Chuankid et al. (2020) recognized a series of pulvinic acid derivatives from liquid cultivation of $P$. spongiosus, whereas some of them had significant antimicrobial and cytotoxic activities. Kumla et al. (2020) were the first to report $P$. spongiosus from northern Thailand and its capability to produce fruiting bodies without a host plant.

Fungicolous fungi are a diverse group of organisms associated with species of different lineages across the fungal kingdom (Sun et al. 2019a, Maurice et al. 2021). They have a wide range of distribution and principally exist as saprotrophs, mycoparasites, symbionts and even neutrals (Sun et al. 2019a). They can be primary consumers with special and often rapid life cycles, which may result in a decrease of certain nutrients in the food webs (Maurice et al. 2021). Several fungicolous fungi have been reported to cause deformation, decomposition or serious diseases in 
cultivated edible and medicinal mushrooms, whose yield and quality are consequently affected (Sun et al. 2019a, b). Therefore, accurate identification and investigation of the taxonomic diversity of fungicolous taxa are essential preliminary steps to understand their associations with related hosts. This would be helpful for quarantine purposes in the mushroom industry and also to devise better disease management methods.

About 1500 fungicolous fungal taxa have been recorded so far, and Aspergillus species are among those (Sun et al. 2019a). Aspergillus species occur worldwide in various habitats and have high economic and social impacts (Hubka et al. 2016). Many species of this genus are used in biotechnology for the production of antibiotics, medicines, enzymes, organic acids and food fermentation agents (Samson et al. 2014). However, they are also known for being able to produce mycotoxins, spoil food and are frequently reported as human, plant and/or animal pathogens (Samson et al. 2014, Hubka et al. 2016).

The identification and classification of Aspergillus species were previously based on phenotypic characters but later, molecular and chemotaxonomic characterizations came into use (Samson et al. 2014). Aspergillus quadrilineatus Thom \& Raper, belongs to series Nidulantes (in section Nidulantes) and shows a homothallic reproduction (Houbraken et al. 2020). Chen et al. (2016) and Hubka et al. (2016) have studied the taxonomy of Aspergillus section Nidulantes in detail. The majority of section Nidulantes species are capable to form a sexual morph and those species were earlier accommodated in Emericella as per the dual name nomenclature system. When the "one fungus: one name" nomenclatural system came into practice, all Emericella species were transferred to Aspergillus (Samson et al. 2014). Aspergillus section Nidulantes species have prominent morphological features, such as biseriate conidiophores with brown-pigmented stipes and ascomata embedded in masses of Hülle cells with often reddish-brown ascospores (Chen et al. 2016, Hubka et al. 2016). All of these species with sexual morph have been reported to have two ascospore crests except in A. quadrilineatus, which has four crests (Chen et al. 2016, Hubka et al. 2016).

During an investigation of fungal taxa associated with commercial and wild mushrooms, we discovered A. quadrilineatus as a fungicolous species on Phlebopus spongiosus grown in a cultivation trial at Mae Fah Luang University, Chiang Rai Province, northern Thailand. This study reports A. quadrilineatus for the first time as a fungicolous species on Phlebopus spongiosus.

\section{Materials \& Methods}

\section{Fungal isolation and morphological characterization}

Phlebopus spongiosus mushrooms covered with white to green powdery mycelium and sporulating structures were collected during a cultivation trial at Mae Fah Luang University in 2019. Fungal mycelia on the mushroom were transferred to potato dextrose agar (PDA) plates using a sterile needle. The plates were incubated at $25{ }^{\circ} \mathrm{C}$ for five days and pure cultures were obtained by subculturing. The micro-morphological examination was conducted using a Nikon ECLIPSE 80i compound microscope and photographs were taken with a Canon 750D digital camera fitted to the microscope. Measurements were made with the Tarosoft (® Image Frame Work version 0.9.7. program and images used for figures were processed with Adobe Photoshop CS6 Extended version 10.0 software (Adobe Systems, USA).

Colony characteristics of cultures were observed and measured after two weeks. Herbarium specimens and living cultures have been deposited in Mae Fah Luang University (MFLU) Herbarium and Culture Collection of Mae Fah Luang University (MFLUCC), Chiang Rai, Thailand, respectively. Faces of Fungi (FoF) number was acquired according to Jayasiri et al. (2015).

\section{DNA extraction, PCR amplification and sequencing}

Fungal isolates were maintained on PDA at $25^{\circ} \mathrm{C}$. Total genomic DNA was extracted from 50 to $100 \mathrm{mg}$ of axenic mycelium scraped from four weeks old cultures. Mycelia were ground to a fine powder with liquid nitrogen and fungal DNA was extracted using the OMEGA E.Z.N.A. ${ }^{\circledR}$ 
Forensic DNA Kit following the manufacturer's instructions. Four loci, namely, the internal transcribed spacer (ITS), partial beta-tubulin (tub2), calmodulin (cam) and DNA directed RNA polymerase II (rpb2) were amplified and sequenced using methods and primers as previously described (Houbraken \& Samson 2011, Samson et al. 2014). The verified PCR fragments were purified according to the company protocols and sequenced by Biomed Co. LTD, Beijing, China. Newly generated nucleotide sequences were deposited in GenBank.

\section{Sequence alignment}

The generated sequences were subjected to nucleotide BLAST search in GenBank (https://blast.ncbi.nlm.nih.gov/Blast.cgi). The BLAST search results, corroborated by initial morphological studies, revealed that our isolates belong to Aspergillus. Related reference sequences were then downloaded from GenBank based on recently published data (Chen et al. 2016, Houbraken et al. 2020) (Table 1). Single gene alignments were obtained using MAFFT v. 7.036 (http://mafft.cbrc.jp/alignment/server/) (Katoh et al. 2019) with default settings and, refined where necessary in BioEdit v. 7.0.5.2 (Hall 1999), as described in Dissanayake et al. (2020).

\section{Phylogenetic analyses}

Maximum likelihood (ML) and Bayesian posterior probability (BYPP) analyses were performed for ITS, $t u b 2$, cam and $r p b 2$ concatenated alignment. The ML trees were generated using RAxML-HPC2 on XSEDE (8.2.8) (Stamatakis et al. 2008, Stamatakis 2014) in the CIPRES Science Gateway V 3.3 platform (Miller et al. 2010). GTRGAMMA was used as the model of evolution and bootstrap support values were obtained by running 1000 replicates. The BYPP analysis was performed with MrBayes v. 3.2.6 (Ronquist \& Huelsenbeck 2003) using a Markov Chain Monte Carlo (MCMC) algorithm according to Dissanayake et al. (2020). The phylograms were visualized with FigTree v1.4.0 program (Rambaut 2011) and reorganized in Microsoft PowerPoint 2010.

Table 1 Strains used for the phylogenetic analyses in this study.

\begin{tabular}{|c|c|c|c|c|c|}
\hline \multirow[t]{2}{*}{ Species } & \multirow[t]{2}{*}{ Collection Number } & \multicolumn{4}{|c|}{ GenBank Accession Number } \\
\hline & & ITS & tub2 & cam & $r p b 2$ \\
\hline $\begin{array}{l}\text { Aspergillus } \\
\text { amethystinus }\end{array}$ & NRRL $4178^{\mathrm{T}}$ & EF652462 & EF652286 & EF652374 & EF652198 \\
\hline A. атоепиs & NRRL $4838^{\mathrm{T}}$ & EF652480 & JN853946 & JN854035 & JN853824 \\
\hline A. angustatus & CBS $273.65^{\mathrm{T}}$ & EU448283 & AY339993 & EU443984 & KU867013 \\
\hline A. askiburgiensis & $\mathrm{CCF} 4716^{\mathrm{T}}$ & LN873939 & LN873952 & LN873965 & LN873984 \\
\hline \multirow[t]{2}{*}{ A. asperescens } & NRRL $4770^{\mathrm{T}}$ & EF652475 & EF652299 & EF652387 & EF652211 \\
\hline & CBS $116.53=$ DTO 020-G8 & KU866661 & KU866888 & KU866689 & KU866940 \\
\hline \multirow[t]{2}{*}{ A. astellatus } & NRRL $2396^{\mathrm{T}}$ & EF652446 & EF652270 & EF652358 & EF652182 \\
\hline & CBS 135.55 & EU448273 & AY339994 & EU443975 & KU866936 \\
\hline A. aurantiobrunneus & NRRL $4545^{\mathrm{T}}$ & EF652465 & EF652289 & EF652377 & EF652201 \\
\hline \multirow[t]{2}{*}{ A. aurantiopurpureus } & $\begin{array}{l}\text { CBS } 140608^{\mathrm{T}}=\text { DTO 060- } \\
\text { A7 }\end{array}$ & KU866588 & KU866824 & KU866711 & KU866966 \\
\hline & $\begin{array}{l}\text { CBS } 140609=\text { DTO 061- } \\
\text { B9 }\end{array}$ & KU866593 & KU866826 & KU866716 & KU866970 \\
\hline \multirow[t]{2}{*}{ A. aureolatus } & NRRL $5126^{\mathrm{T}}$ & EF652501 & EF652325 & EF652413 & EF652237 \\
\hline & $\begin{array}{l}\text { CBS } 138434=\text { DTO 131- } \\
\text { G5 }\end{array}$ & KU866663 & KU866898 & KU866726 & KU866985 \\
\hline A. austroafricanus & NRRL $233^{\mathrm{T}}$ & JQ301891 & JN853963 & JN854025 & JN853814 \\
\hline A. botswanensis & CBS $314.89^{\mathrm{T}}=$ DTO 047-I4 & KU866572 & KU866812 & KU866695 & KU866949 \\
\hline \multirow[t]{2}{*}{ A. caespitosus } & NRRL $1929^{\mathrm{T}}$ & EF652428 & EF652252 & EF652340 & EF652164 \\
\hline & DTO 325-C1 & KU866669 & KU866907 & KU866789 & KU867054 \\
\hline
\end{tabular}


Table 1 Strains used for the phylogenetic analyses in this study.

\begin{tabular}{|c|c|c|c|c|c|}
\hline \multirow[t]{2}{*}{ Species } & \multirow[t]{2}{*}{ Collection Number } & \multicolumn{4}{|c|}{ GenBank Accession Number } \\
\hline & & ITS & tub2 & cam & $r p b 2$ \\
\hline A. corrugatus & CBS $191.77^{\mathrm{T}}=$ DTO 047-I9 & KU866574 & KU866814 & KU866696 & KU866951 \\
\hline A. creber & NRRL $58592^{\mathrm{T}}$ & JQ301889 & JN853980 & JN854043 & JN853832 \\
\hline \multirow[t]{2}{*}{ A. croceiaffinis } & CCF $6035^{\mathrm{T}}$ & MK713538 & MK695645 & MK695656 & MK695667 \\
\hline & CCF 6036 & MK713537 & MK695644 & MK695655 & MK695666 \\
\hline A. croceus & $\mathrm{CCF} 4405^{\mathrm{T}}$ & LN873931 & LN873944 & LN873957 & LN873976 \\
\hline A. cvjetkovicii & NRRL $227^{\mathrm{T}}$ & EF652440 & EF652264 & EF652352 & EF652176 \\
\hline \multirow[t]{2}{*}{ A. desertorum } & NRRL $5921^{\mathrm{T}}$ & EF652505 & EF652329 & EF652417 & EF652241 \\
\hline & CBS 654.73 = DTO 047-I1 & KU866571 & KU866811 & KU866694 & KU866948 \\
\hline A. dipodomyus & NRRL $66273^{\mathrm{T}}$ & MK713535 & MK695642 & MK695653 & MK695664 \\
\hline \multirow[t]{2}{*}{ A. dromiae } & $\begin{array}{l}\text { CBS } 140633^{\mathrm{T}}=\text { DTO 059- } \\
\text { H5 }\end{array}$ & KU866580 & KU866885 & KU866703 & KU866958 \\
\hline & CBS 140639 & KU866672 & KU866909 & KU866792 & KU867057 \\
\hline \multirow[t]{2}{*}{ A. falconensis } & CBS $271.91^{\mathrm{T}}=$ DTO 048-A2 & KU866575 & KU866815 & KU866697 & KU866952 \\
\hline & CBS 989.72 = DTO 048-A3 & KU866576 & KU866816 & KU866698 & KU866953 \\
\hline \multirow[t]{2}{*}{ A. filifer } & CBS $113636^{\mathrm{T}}$ & EU448277 & EF428372 & EU443973 & KU866932 \\
\hline & $\begin{array}{l}\text { CBS } 128790=\text { IFM } 54232= \\
\text { DTO 098-H9 }\end{array}$ & AB248963 & AB248305 & KU866724 & KU866983 \\
\hline \multirow[t]{2}{*}{ A. foveolatus } & CBS $279.81^{\mathrm{T}}$ & KX423658 & KX423622 & KX423635 & KU867034 \\
\hline & CBS 542.83 = DTO 319-I2 & KU866615 & KU866857 & KU866754 & KU867016 \\
\hline A. fructus & NRRL $239^{\mathrm{T}}$ & EF652449 & EF652273 & EF652361 & EF652185 \\
\hline A. fruticulosus & NRRL $4903^{\mathrm{T}}$ & EF652483 & EF652307 & EF652395 & EF652219 \\
\hline A. griseoaurantiacus & $\begin{array}{l}\text { CBS } 138191 \mathrm{~T}=\text { DTO 267- } \\
\text { D8 }\end{array}$ & KJ775553 & KJ775086 & KJ775357 & KU866988 \\
\hline A. hongkongensis & HKU49 & AB987907 & LC000552 & LC000565 & LC000578 \\
\hline A. incahuasiensis & NRRL $66825^{\mathrm{T}}$ & MH473585 & MH476273 & MH476276 & MH476279 \\
\hline \multirow[t]{2}{*}{ A. israelensis } & CBS $140627^{\mathrm{T}}$ & KU866677 & KU866915 & KU866797 & KU867062 \\
\hline & CBS 140628 & KU866678 & KU866916 & KU866798 & KU867063 \\
\hline \multirow[t]{2}{*}{ A. jaipurensis } & CBS $952.97^{\mathrm{T}}=$ DTO 320-A9 & KU866623 & AY339988 & KU866761 & KU867024 \\
\hline & CBS 100253 & KU866675 & KU866913 & KU866795 & KU867060 \\
\hline A. jensenii & NRRL $58600^{\mathrm{T}}$ & JQ301892 & JN854007 & JN854046 & JN853835 \\
\hline A. latilabiatus & CBS $426.93^{\mathrm{T}}=$ DTO $320-\mathrm{B} 2$ & KU866624 & KU866864 & KU866762 & KU867025 \\
\hline \multirow[t]{2}{*}{ A. longistipitatus } & CCF $5788^{\mathrm{T}}$ & MK713534 & MK695641 & MK695652 & MK695663 \\
\hline & CCF 6117 & MK713533 & MK695640 & MK695651 & MK695662 \\
\hline A. miraensis & $\mathrm{CBS} 140625^{\mathrm{T}}=\mathrm{DTO} 323-\mathrm{B} 2$ & KU866642 & KC342577 & KU866780 & KU867045 \\
\hline A. multicolor & NRRL $4775^{\mathrm{T}}$ & EF652477 & EF652301 & EF652389 & EF652213 \\
\hline A. mulundensis & $\begin{array}{l}\text { CBS } 140610 \mathrm{~T}=\text { DTO 316- } \\
\text { C9 }\end{array}$ & KU866604 & KU866833 & KU866729 & KU866989 \\
\hline A. navahoensis & NRRL $13002^{\mathrm{T}}$ & EF652424 & EF652248 & EF652336 & EF652160 \\
\hline \multirow[t]{2}{*}{ A. nidulans } & NRRL 187 & EF652427 & EF652251 & EF652339 & EF652163 \\
\hline & DTO 065-F9 & KU866599 & KU866831 & KU866722 & KU866977 \\
\hline \multirow[t]{2}{*}{ A. olivicola } & CBS $119.37^{\mathrm{T}}$ & EU448268 & AY339996 & EU443986 & KU866923 \\
\hline & CBS 597.65 & EU448267 & AY339997 & EU443985 & KU866935 \\
\hline A. omanensis & CBM FA-700 ${ }^{\mathrm{T}}=$ IFM 54275 & - & AB248347 & AB524047 & - \\
\hline \multirow[t]{2}{*}{ A. pachycristatus } & IFM $55265^{\mathrm{T}}$ & - & AB375875 & AB524062 & - \\
\hline & DTO 060-A3 & KU866585 & KU866821 & KU866708 & KU866963 \\
\hline A. pepii & AV11051B_IX ${ }^{\mathrm{T}}$ & KU613368 & KU613371 & KU613365 & - \\
\hline
\end{tabular}


Table 1 Strains used for the phylogenetic analyses in this study.

\begin{tabular}{|c|c|c|c|c|c|}
\hline \multirow[t]{2}{*}{ Species } & \multirow[t]{2}{*}{ Collection Number } & \multicolumn{4}{|c|}{ GenBank Accession Number } \\
\hline & & ITS & tub2 & cam & $r p b 2$ \\
\hline \multirow[t]{2}{*}{ A. pluriseminatus } & CBS $100523^{\mathrm{T}}$ & KU866566 & AY339989 & EU443988 & KU866937 \\
\hline & CBS $102705=$ DTO 010-I8 & KU866565 & KU866806 & KU866686 & KU866926 \\
\hline A. protuberus & NRRL $3505^{\mathrm{T}}$ & EF652460 & EF652284 & EF652372 & EF652196 \\
\hline \multirow{2}{*}{$\begin{array}{l}\text { A. } \\
\text { purpureocrustaceus }\end{array}$} & $\mathrm{CMV008B}^{\mathrm{T}}$ & MK450653 & MK451138 & MK451515 & MK450806 \\
\hline & CMV008B1 & MK450652 & MK451137 & MK451514 & MK450805 \\
\hline A. purpureus & NRRL $6133^{\mathrm{T}}$ & EF652506 & EF652330 & EF652418 & EF652242 \\
\hline A. puulaauensis & NRRL $35641^{\mathrm{T}}$ & JQ301893 & JN853979 & JN854034 & JN853823 \\
\hline \multirow[t]{2}{*}{ A. qinqixianii } & $\begin{array}{l}\text { CBS } 128788=\text { CMB-FA-866 } \\
\mathrm{T}\end{array}$ & KU866600 & AB524360 & AB524051 & KU866980 \\
\hline & CBS $128789=$ DTO 098-H7 & KU866601 & KU866894 & KU866723 & KU866981 \\
\hline \multirow[t]{15}{*}{ A. quadrilineatus } & NRRL $201^{\mathrm{T}}$ & EF652433 & EF652257 & EF652345 & EF652169 \\
\hline & CBS $937.73=$ DTO 020-I9 & KU866568 & KU866808 & KU866691 & KU866942 \\
\hline & CBS $119.55=$ NRRL 2394 & EF652444 & AY573549 & AB476805 & KU866945 \\
\hline & $\begin{array}{l}\text { CBS } 493.65=\text { IMI } 139280= \\
\text { IFM } 48171\end{array}$ & KU866570 & AB243111 & AB524048 & KU866947 \\
\hline & CBS 125.55 = DTO 048-A8 & KU866577 & KU866817 & KU866699 & KU866954 \\
\hline & CBS 113684 = DTO 319-F9 & KU866607 & KU866850 & KU866746 & KU867007 \\
\hline & CBS $118.51=$ DTO 319-G2 & KU866609 & KU866852 & KU866748 & KU867009 \\
\hline & CBS $467.88=$ DTO 320-C3 & KU866630 & KU866869 & KU866768 & KU867032 \\
\hline & DTO 322-D3 & KU866634 & KU866871 & KU866772 & KU867037 \\
\hline & DTO 322-I8 & KU866639 & KU866874 & KU866777 & KU867042 \\
\hline & CBS 126215 & KU866674 & KU866912 & KU866794 & KU867059 \\
\hline & IFM 54233 = CBM-FA-833 & - & AB243110 & AB524040 & - \\
\hline & CBS $853.96=$ DTO 320-A8 & KU866622 & KU866863 & KU866760 & KU867023 \\
\hline & MFLUCC 21-0165 & OL615082 & OL625674 & - & - \\
\hline & MFLUCC 21-0166 & OL615089 & OL625675 & - & - \\
\hline \multirow[t]{2}{*}{ A. recurvatus } & CBS $496.65^{\mathrm{T}}$ & EF652482 & EF652306 & EF652394 & EF652218 \\
\hline & CBS $126259=$ DTO 195-D8 & KU866603 & KU866832 & KU866727 & KU866986 \\
\hline \multirow[t]{2}{*}{ A. rugulosus } & NRRL $206^{\mathrm{T}}$ & EF652434 & EF652258 & EF652346 & EF652170 \\
\hline & CBS 130.48 = DTO 319-H5 & KU866611 & KU866853 & KU866750 & KU867011 \\
\hline \multirow[t]{2}{*}{ A. savannensis } & $\begin{array}{l}\text { CBS } 140607^{\mathrm{T}}=\text { DTO 059- } \\
\text { H6 }\end{array}$ & KU866581 & KU866818 & KU866704 & KU866959 \\
\hline & CBS $126213=$ DTO 061-B8 & KU866592 & KU866825 & KU866715 & KU866969 \\
\hline A. spelunceus & NRRL $4989^{\mathrm{T}}$ & EF652490 & EF652314 & EF652402 & EF652226 \\
\hline \multirow[t]{2}{*}{ A. spinulosporus } & CBS $120.55^{\mathrm{T}}=$ NRRL 2395 & EF652445 & AY573553 & EF652357 & EF652181 \\
\hline & DTO 065-F7 & KU866598 & KU866830 & KU866721 & KU866976 \\
\hline \multirow[t]{2}{*}{ A. stella-maris } & $\begin{array}{l}\text { CBS } 113638^{\mathrm{T}}=\text { DTO 011- } \\
\text { A2 }\end{array}$ & EU448269 & KU866886 & EU443978 & KU866929 \\
\hline & CBS $113639=$ DTO 011-A3 & EU448270 & EF428367 & KU866687 & KU866930 \\
\hline \multirow[t]{2}{*}{ A. stellatus } & NRRL $1858^{\mathrm{T}}$ & EF652426 & EF652250 & EF652338 & EF652162 \\
\hline & DTO 059-H2 & KU866579 & KU866889 & KU866702 & KU866957 \\
\hline A. stelliformis & CCF $5375^{\mathrm{T}}$ & MK713531 & MK695638 & MK695649 & MK695660 \\
\hline A. stercorarius & CBS $428.93^{\mathrm{T}}=$ DTO $320-\mathrm{B} 3$ & KU866625 & KU866865 & KU866763 & KU867026 \\
\hline \multirow[t]{2}{*}{ A. striatus } & NRRL $4699^{\mathrm{T}}$ & EF652470 & EF652294 & EF652382 & EF652206 \\
\hline & CBS $866.70=$ DTO 320-A7 & KU866621 & KU866862 & KU866759 & KU867022 \\
\hline
\end{tabular}


Table 1 Strains used for the phylogenetic analyses in this study.

\begin{tabular}{|c|c|c|c|c|c|}
\hline \multirow[t]{2}{*}{ Species } & \multirow[t]{2}{*}{ Collection Number } & \multicolumn{4}{|c|}{ GenBank Accession Number } \\
\hline & & ITS & tub2 & cam & $r p b 2$ \\
\hline \multirow[t]{2}{*}{ A. sublatus } & $\begin{array}{l}\text { CBS } 492.65^{\mathrm{T}}=\text { DTO } 047-\mathrm{H} 2 \\
=\mathrm{IFM} 42011\end{array}$ & KF465768 & AB248334 & KU866693 & KU866946 \\
\hline & CBS 140630 & KU866683 & KU866920 & KU866804 & KU867069 \\
\hline A. subversicolor & NRRL 58999 & JQ301894 & JN853970 & JN854010 & JN853799 \\
\hline A. sulphureoviridis & CBS $140626^{\mathrm{T}}$ & KU866673 & KU866911 & KU866793 & KU867058 \\
\hline A. sydowii & NRRL 250 & EF652450 & EF652274 & EF652362 & EF652186 \\
\hline A. tabacinus & NRRL $4791^{\mathrm{T}}$ & EF652478 & EF652302 & EF652390 & EF652214 \\
\hline A. tennesseensis & NRRL $13150^{\mathrm{T}}$ & JQ301895 & JN853976 & JN854017 & JN853806 \\
\hline A. tumidus & FMR $15743^{\mathrm{T}}$ & LT903691 & LT903682 & LT903685 & LT903688 \\
\hline \multirow[t]{2}{*}{ A. undulates } & CBS $261.88^{\mathrm{T}}$ & EU448275 & EF428363 & EU443989 & KU866928 \\
\hline & DTO 322-B2 & KU866633 & KU866901 & KU866771 & KU867036 \\
\hline \multirow[t]{2}{*}{ A. unguis } & NRRL $2393^{\mathrm{T}}$ & EF652443 & EF652267 & EF652355 & EF652179 \\
\hline & CBS 595.65 = DTO 319-I5 & KU866616 & KU866858 & FN594611 & KU867017 \\
\hline \multirow[t]{2}{*}{ A. ustus } & CBS 261.67T & $\begin{array}{l}\text { NR_13128 } \\
4\end{array}$ & EF591727 & EF591719 & - \\
\hline & NRRL 275 & EF652455 & EF652279 & EF652367 & EF652191 \\
\hline \multirow[t]{2}{*}{ A. varians } & NRRL $4793^{\mathrm{T}}$ & EF652479 & EF652303 & EF652391 & EF652215 \\
\hline & DTO 063-I1 & KU866597 & KX423620 & KU866720 & - \\
\hline A. venenatus & NRRL $13147^{\mathrm{T}}$ & JQ301896 & JN854003 & JN854014 & JN853803 \\
\hline A. venezuelensis & CBS $868.97^{\mathrm{T}}$ & AJ874119 & AY339998 & EU443977 & KU866931 \\
\hline A. versicolor & NRRL 238 & EF652442 & EF652266 & EF652354 & EF652178 \\
\hline \multirow[t]{2}{*}{ A. violaceus } & NRRL $2240^{\mathrm{T}}$ & EF652438 & EF652262 & EF652350 & EF652174 \\
\hline & CBS 293.93 & EU448279 & EF428374 & EU443987 & KU866927 \\
\hline A. viridicatenatus & CBS $140629^{\mathrm{T}}=$ DTO $325-\mathrm{F} 4$ & KU866682 & KX423621 & KU866802 & KU867067 \\
\hline
\end{tabular}

Note: The strain numbers of type strains are superscripted with $\mathrm{T}$ and newly generated strains are in red.

\section{Results}

\section{Phylogenetic analyses}

The combined ITS, $t u b 2$, cam and $r p b 2$ matrix comprised 122 strains including selected taxa in Aspergillus section Nidulantes and Aspergillus ustus (which belongs to Aspergillus section Usti) as the outgroup (Fig. 1). The single gene ML trees (Figs 1 and 2 in supplementary section) for ITS and $t u b 2$ showed different topologies compared to the concatenated alignment. The concatenated alignment resulted in similar topologies for both ML and BYPP. The value of the average standard deviation of the split frequencies of BYPP analysis was 0.009970. The best scoring RAxML tree is shown in Figure 1 with a final ML optimization likelihood value of -28316.145006 . The matrix had 1261 distinct alignment patterns, with $11.16 \%$ of undetermined characters or gaps. Estimated base frequencies were as follows: $\mathrm{A}=0.236496, \mathrm{C}=0.270741, \mathrm{G}=0.262933, \mathrm{~T}=0.229830$; substitution rates $\mathrm{AC}=1.220496, \mathrm{AG}=4.721561, \mathrm{AT}=1.555801, \mathrm{CG}=0.960300, \mathrm{CT}=$ 6.996935, GT $=1.000000$; gamma distribution shape parameter $\alpha=0.251927$. Our isolates (MFLUCC 21-0165 and MFLUCC 21-0166) clustered in the Aspergillus quadrilineatus clade. 


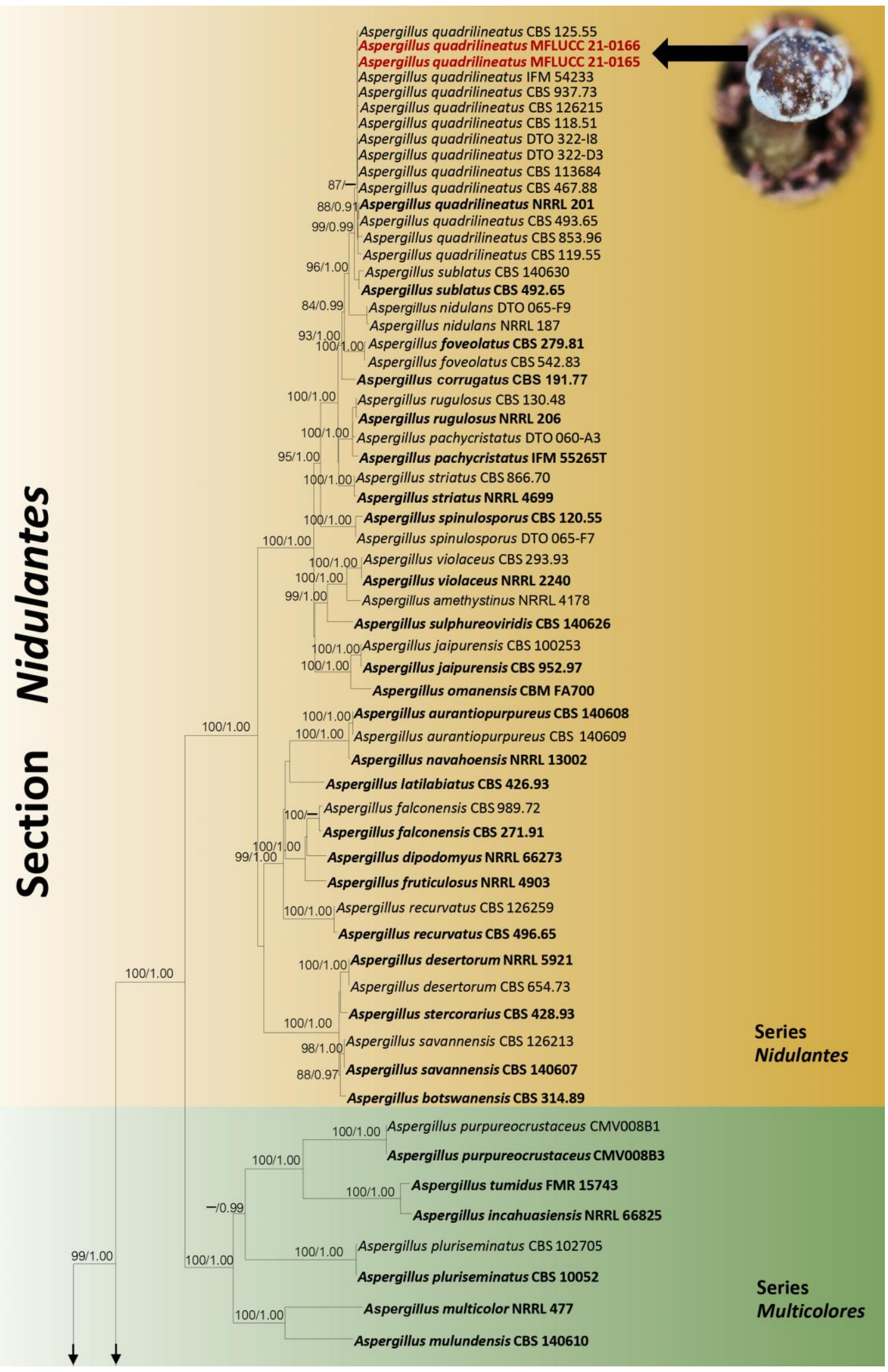

Fig. 1 - The best scoring RAxML tree based on the analysis of ITS, tub2, cam and $r p b 2$ combined dataset. Bootstrap support values for ML values equal to or $>70 \%$ and BYPP values equal to or $>0.95$ are shown as ML/BYPP near the nodes. The isolates used for the present study are shown in red bold. The type-derived sequences are given in black bold. The tree is rooted with Aspergillus ustus (NRRL 275 and CBS 261.67). The scale bar represents the expected number of nucleotide substitutions per site. 


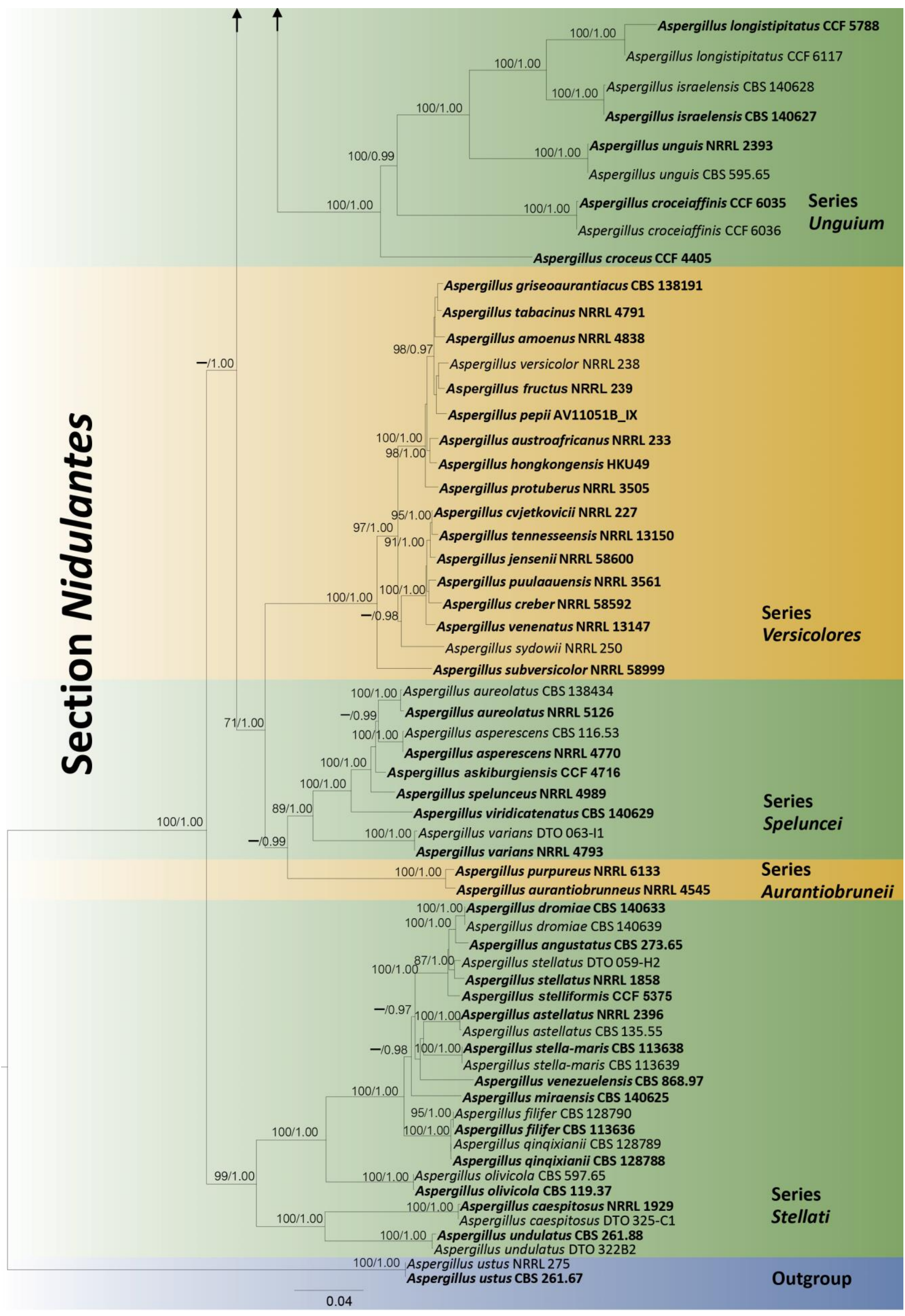

Fig. 1 - Continued. 


\section{Taxonomic description}

Aspergillus quadrilineatus Thom \& Raper, Mycologia 31: 660. (1939).

Figs 2, 3

Index Fungorum number: IF 275888

Facesoffungi Number: FoF 10610

Fungicolous on the fruiting body of Phlebopus spongiosus Pham and Har. Takah. Sexual morph on PDA: Ascomata 190-310 $\times 160-300 \mu \mathrm{m}(\bar{x}=227.6 \times 221.6 \mu \mathrm{m}, \mathrm{n}=5)$, cleistothecial, developing throughout the colony, reddish-brown, globose to subglobose, surrounded by numerous Hülle cells; Hülle cells $12.5-23 \times 11.5-22 \mu \mathrm{m}(\bar{x}=17.5 \times 17.3 \mu \mathrm{m}, \mathrm{n}=30)$, hyaline to brown, globose to ovoid. Asci 9-11 $\times 8-11 \mu \mathrm{m}(\bar{x}=10.1 \times 9.9 \mu \mathrm{m}, \mathrm{n}=15), 8$-spored, globose to subglobose. Ascospores 3-4 $\times 3-4 \mu \mathrm{m}(\bar{x}=3.8 \times 3.4 \mu \mathrm{m}, \mathrm{n}=40)$, orange to reddish-brown, globose to subglobose in surface view, spore bodies smooth, lenticular in lateral view, with two plaited equatorial crests about $0.5-1 \mu \mathrm{m}$ in width paralleled by a secondary narrower pair which are sometimes indistinct, crests are entire, defective or with irregular protuberance. Asexual morph on host: Mycelium powdery mass, white to green; sporulation light green to dark green. PDA: Conidiophores 80-165 × 3-6 $\mu \mathrm{m}(\bar{x}=122.2 \times 4.3 \mu \mathrm{m}, \mathrm{n}=15)$ macronematous, mononematous, erect, simple, with septate to aseptate stipes, pale brown; Vesicles 9-12 $\mu \mathrm{m}(\bar{x}=10.6 \mu \mathrm{m}, \mathrm{n}=15)$ wide, pale brown, globose to subglobose; Metulae 3-4.5 $\times 1-2 \mu \mathrm{m}(\bar{x}=3.7 \times 1.5 \mu \mathrm{m}, \mathrm{n}=20)$ hyaline; Phialides 4.5-6 × 1-2 $\mu \mathrm{m}(\bar{x}=5.7 \times 1.4 \mu \mathrm{m}, \mathrm{n}=20)$ hyaline, flask-shaped; Conidia 2.5-4 $\times 2-4 \mu \mathrm{m}(\bar{x}=3.2 \times 3.3 \mu \mathrm{m}, \mathrm{n}=50)$ slightly echinulate, globose to subglobose, hyaline to light green.

Colony characters: on PDA, $25^{\circ} \mathrm{C}, 14$ days: Colonies moderately deep, marginally sulcate; margins slightly irregular; mycelium buff and white, later turns to reddish-brown; texture floccose; sporulation sparse; soluble pigments absent; exudates clear droplets; reverse dark brown.

Material examined: THAILAND, Chiang Rai Province, isolated using the mycelium growing on the fruiting body of Phlebopus spongiosus cultivated in soil, 29 November 2019, Bhavesh Raghoonundon, AJ 044 (inactive dry culture MFLU 21-0261), living cultures MFLUCC 21-0165 and MFLUCC 21-0166.

GenBank accession numbers: MFLUCC 21-0165: ITS: OL615082; tub2: OL625674. MFLUCC 21-0166: ITS: OL615089; tub2: OL625675.

Notes: Aspergillus quadrilineatus strains MFLUCC 21-0165 and MFLUCC 21-0166 reported in this study share similar sexual and asexual morphologies with those observed from the culture of the neotype of A. quadrilineatus (NRRL201) described by Chen et al. (2016). The ascomata, Hülle cells, ascospores, vesicles, metulae and phialides of MFLU 21-0261 are comparatively smaller than those of A. quadrilineatus described in Chen et al. (2016) as shown in Table 2. These dimensional differences occurred probably due to the variations of agar medium used to obtain the culture.

During this study, all four loci namely, ITS, tub2, cam and $r p b 2$ were amplified and sequenced using methods and primers as previously described in Houbraken \& Samson (2011) and Samson et al. (2014). However, only the ITS and $t u b 2$ sequence results were obtained successfully. There were no base pair differences of ITS and tub2 sequences between the neotype of $A$. quadrilineatus (NRRL 201) described by Chen et al. (2016) and Aspergillus quadrilineatus strains MFLUCC 21-0165 and MFLUCC 21-0166 reported in this study.

Table 2: The comparison of the sizes of fungal structures.

\begin{tabular}{lll}
\hline Fungal structure & Chen et al. (2016) & This study \\
\hline Ascomata & $100-700 \mu \mathrm{m}$ & $190-310 \times 160-300 \mu \mathrm{m}$ \\
Hülle cells & $10-24 \mu \mathrm{m}$ & $12.5-23 \times 11.5-22 \mu \mathrm{m}$ \\
Ascospores & $4-4.5 \times 3-4.5 \mu \mathrm{m}$ & $3-4 \times 3-4 \mu \mathrm{m}$ \\
Vesicles & $10-13 \mu \mathrm{m}$ & $9-12 \mu \mathrm{m}$ \\
Metulae & $5-7 \times 2-4.5 \mu \mathrm{m}$ & $3-4.5 \times 1-2 \mu \mathrm{m}$ \\
Phialides & $5-7 \times 2-4 \mu \mathrm{m}$ & $4.5-6 \times 1-2 \mu \mathrm{m}$ \\
\hline
\end{tabular}




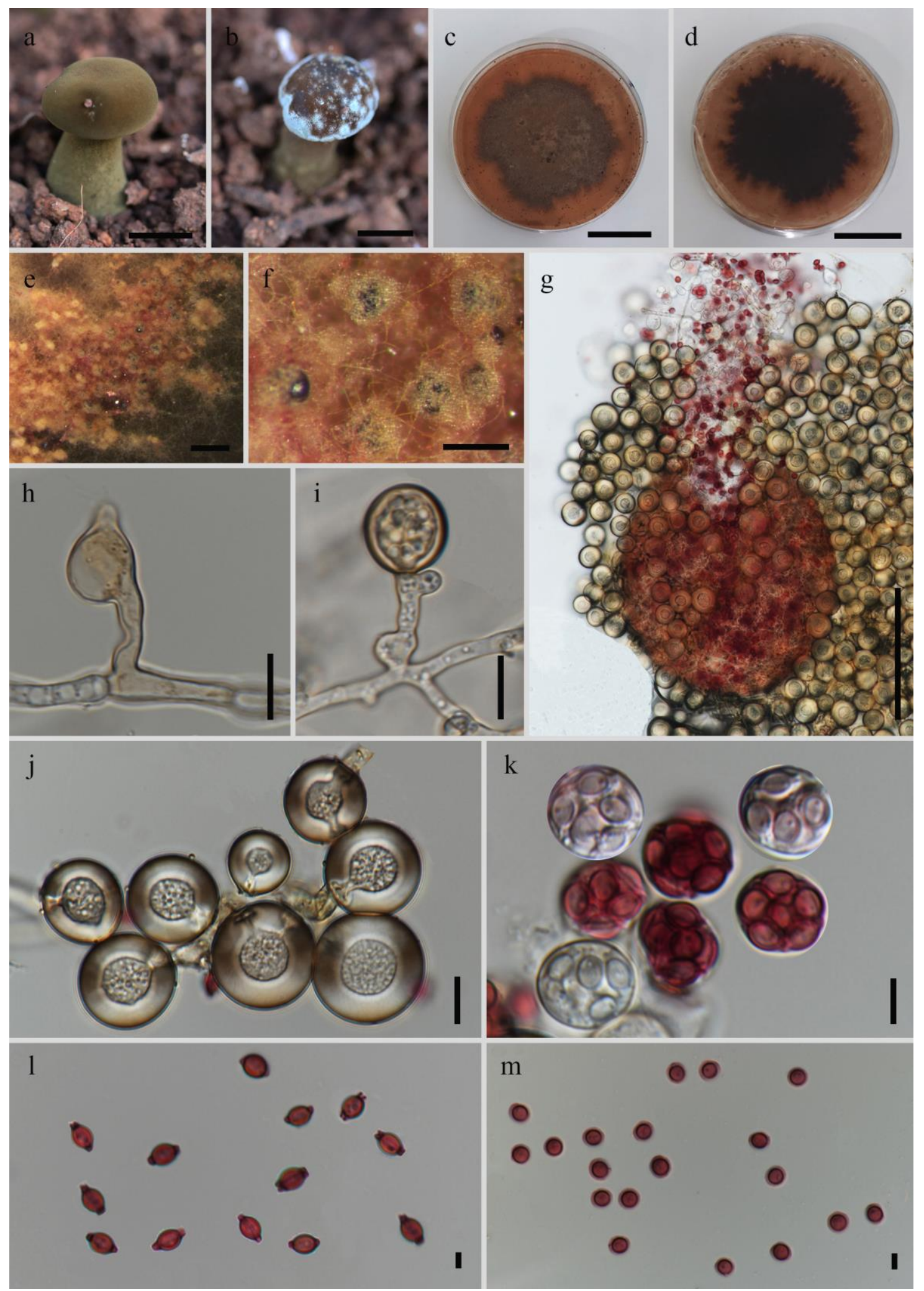

Fig. 2 - Sexual morph of Aspergillus quadrilineatus (MFLU 21-0261). a Healthy mushroom, Phlebopus spongiosus growing in soil. b The mushroom affected by Aspergillus quadrilineatus. c, $\mathbf{d}$ Colony on PDA. e, $\mathbf{f}$ Cleistothecial ascomata formation in the colony on PDA. $\mathbf{g}$ Cleistothecium covered with Hülle cells. h, i Formation of Hülle cells. $\mathbf{j}$ Hülle cells. $\mathbf{k}$ Immature and mature asci. I Side view of ascospores. $\mathbf{m}$ Surface view of ascospores. Scale bars: $a, b=2 \mathrm{~cm}$, $\mathrm{c}, \mathrm{d}=30 \mathrm{~mm}, \mathrm{e}=300 \mu \mathrm{m}, \mathrm{f}=500 \mu \mathrm{m}, \mathrm{g}=100 \mu \mathrm{m}, \mathrm{h}-\mathrm{j}=10 \mu \mathrm{m}, \mathrm{k}=5 \mu \mathrm{m}, \mathrm{l}, \mathrm{m}=3 \mu \mathrm{m}$. 


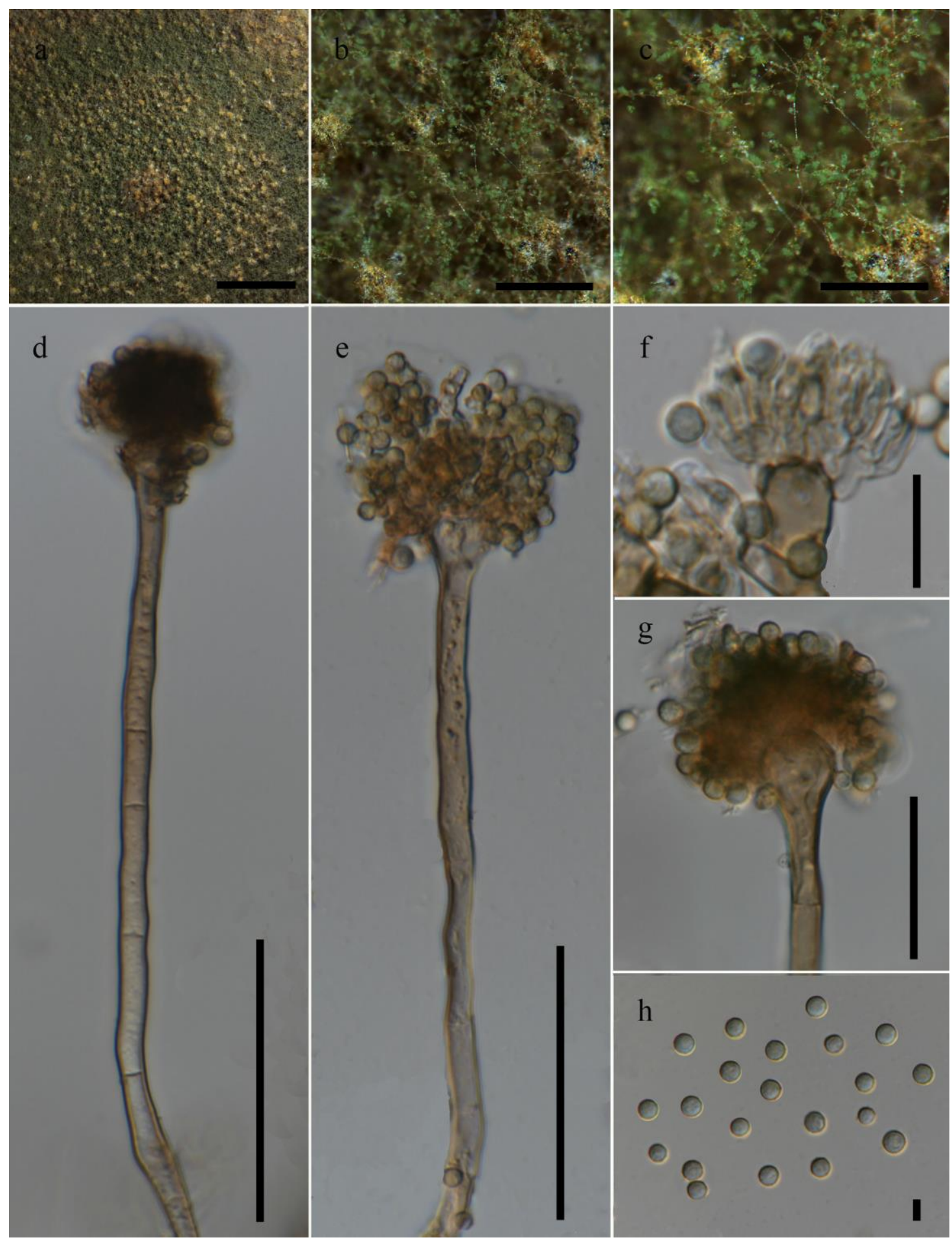

Fig. 3 - Asexual morph of Aspergillus quadrilineatus (MFLU 21-0261). a Colony of Aspergillus on PDA. b, $\mathbf{c}$ Sporulation on PDA. d, e Mononematous conidiophores with conidia. $\mathbf{f}, \mathbf{g}$ Apex of conidiogenous cell. $\mathbf{h}$ Conidia. Scale bars: $\mathrm{a}=5 \mathrm{~mm}, \mathrm{~b}=1 \mathrm{~mm}, \mathrm{c}=500 \mu \mathrm{m}, \mathrm{d}, \mathrm{e}=50 \mu \mathrm{m}, \mathrm{f}=10$ $\mu \mathrm{m}, \mathrm{g}=25 \mu \mathrm{m}, \mathrm{h}=3 \mu \mathrm{m}$.

\section{Discussion}

In the present study, we isolated a fungus growing as a contamination on a mushroom. The morphological and phylogenetic evidence revealed that the fungus growing on mushroom was $A$. quadrilineatus. The single gene phylogenies for ITS and tub2 were not much helpful to resolve the relationships among the species in Aspergillus section Nidulantes.

Previously, different Aspergillus species have been reported associated with mushrooms (Table 3). Herein, A. quadrilineatus is reported for the first time as a fungicolous fungus on 
Phlebopus spongiosus in northern Thailand. It has also been formerly documented to be associated with Elaeis guineensis, Lens culinaris, Lupinus termis, Pisum sativum and human infections (Chadiesh et al. 2017, Seyedmousavi et al. 2018, Farr \& Rossman 2021). Aspergillus quadrilineatus is a soil fungus commonly isolated in tropical countries (Polacheck et al. 1992). Therefore, most probably the primary inoculation of A. quadrilineatus spores may be from the soil used for cultivation or the surrounding air. This ultimately led to fungal contamination on the mushroom cultivation.

Aspergillus section Nidulantes are able to produce mycotoxins such as aflatoxins, asteltoxins, emestrin, fumitremorgins, paxillin, sterigmatocystin and other extrolites useful as drugs or drug lead candidates such as calbistrins, echinocandins, mulundocandins, terrain, variecolins and varitriols (Chen et al. 2016). Sterigmatocystin is a carcinogenic mycotoxin, while aflatoxin has become a major problem in the agricultural and food industries (Nieto et al. 2018). Aspergillus quadrilineatus has been reported to produce extrolites such as asperthecin, asperugins, echinocandins, emestrin, emericellin, emindols, quadrilineatin, shamixanthone, sterigmatocystin and violaceols. (Chen et al. 2016).

Phlebopus spongiosus is an edible fungus (Kumla et al. 2020). Aspergillus quadrilineatus growing on $P$. spongiosus as a fungicolous fungi is able to produce carcinogenic mycotoxins such as sterigmatocystin. Therefore, this may be a food safety issue and it is a challenge to overcome during the cultivation of $P$. spongiosus on soil. Furthermore, Aspergillus quadrilineatus would be able to cause both yield and quality losses in commercial cultivation of $P$. spongiosus on soil resulting economic losses for mushroom farmers. As we were able to isolate A. quadrilineatus from a live fruiting body, it can be potentially pathogenic on $P$. spongiosus; future studies are required with pathogenicity tests for confirmation. The screening of potential biocontrol agents to control $A$. quadrilineatus on $P$. spongiosus also would be important in commercial scale cultivation of $P$. spongiosus on soil.

Table 3 Aspergillus species recorded to be associated with mushrooms worldwide.

\begin{tabular}{|c|c|c|c|c|}
\hline Species & Substrate & Host & Country & Reference \\
\hline Aspergillus fumigatus & $\begin{array}{l}\text { Compost, Straw } \\
\text { and substrate }\end{array}$ & $\begin{array}{l}\text { Agaricus bisporus; } \\
\text { Pleurotus ostreatus }\end{array}$ & Sri Lanka & $\begin{array}{l}\text { Wickremasinghe et al. } \\
\text { (1998) }\end{array}$ \\
\hline A. polyporicola & Fruiting body & Earliella scabrosa & USA & Sun et al. (2019a) \\
\hline A.pseudoglaucus & Fruiting body & wild Helvella sp. & China & Sun et al. (2019b) \\
\hline A. pulverulentus & $\begin{array}{l}\text { Air of } \\
\text { greenhouse }\end{array}$ & Lentinula edodes & Korea & Kwon et al. (2015) \\
\hline A. quadrilineatus & Fruiting body & Phlebopus spongiosus & Thailand & Present study \\
\hline A. tubingensis & $\begin{array}{l}\text { Fruiting body } \\
\text { and compost }\end{array}$ & Cyclocybe aegerita & Korea & Choi et al. (2010) \\
\hline
\end{tabular}

\section{Acknowledgements}

The authors would like to thank Mae Fah Luang University grant, "Identification of fungicolous fungi in northern Thailand and their biological control" (Grant number 651B01010). The authors would like to thank Mae Fah Luang University, Center of Excellence in Fungal Research and Mushroom Research Foundation, Thailand for the valuable support provided for research. Mr. Bhavesh Raghoonundon and Dr. Kawporn Sussangkarnis are thanked for their valuable support for this study.

\section{References}

Chadiesh N, Jishnu BT, Tupaki-Sreepurna A, Ramanan R et al. 2017 - Aspergillus quadrilineatus infection in an elderly debilitated patient. Journal of The Academy of Clinical Microbiologists 19, 62-65. 
Chen AJ, Frisvad JC, Sun BD, Varga J et al. 2016 - Aspergillus section Nidulantes (formerly Emericella): polyphasic taxonomy, chemistry and biology. Studies in mycology 84, 1-118.

Choi IY, Choi JN, Sharma PK, Lee WH. 2010 - Isolation and identification of mushroom pathogens from Agrocybe aegerita. Mycobiology 38, 310-315.

Chuankid B, Schrey H, Thongbai B, Raspé O et al. 2020 - Secondary metabolites of Phlebopus species from Northern Thailand. Mycological Progress 19, 1525-1536.

Dissanayake AJ, Maharachchikumbura SSN, Bhunjun CS, Liu JK. 2020 - Applied aspects of methods to infer phylogenetic relationships amongst fungi. Mycosphere 11, 2652-2676.

Farr DF, Rossman AY. 2021 - Fungal Databases, U.S. National Fungus Collections, ARS, USDA. Available at: https://nt.ars-grin.gov/fungaldatabases/ (Accessed on July 14, 2021).

Hall TA. 1999 - BioEdit: a user-friendly biological sequence alignment editor and analysis program for Windows 95/98/NT. Nucleic Acids Symposium Series 41, 95-98.

Houbraken J, Kocsubé S, Visagie CM, Yilmaz N et al. 2020 - Classification of Aspergillus, Penicillium, Talaromyces and related genera (Eurotiales): An overview of families, genera, subgenera, sections, series and species. Studies in mycology 95, 5-169.

Houbraken JAMP, Samson RA. 2011 - Phylogeny of Penicillium and the segregation of Trichocomaceae into three families. Studies in mycology 70, 1-51.

Hubka V, Nováková A, Peterson SW, Frisvad JC et al. 2016 - A reappraisal of Aspergillus section Nidulantes with descriptions of two new sterigmatocystin-producing species. Plant Systematics and Evolution 302, 1267-1299.

Jayasiri SC, Hyde KD, Ariyawansa HA, Bhat J et al. 2015 - The Faces of Fungi database: fungal names linked with morphology, phylogeny and human impacts. Fungal Diversity 74: 3-18.

Katoh K, Rozewicki J, Yamada KD. 2019 - MAFFT online service: multiple sequence align-ment, interactive sequence choice and visualization. Briefings in Bioinformatics 20: 1160-1166.

Kumla J, Suwannarach N, Lumyong S. 2020 - A New Report on Edible Tropical Bolete, Phlebopus spongiosus in Thailand and Its Fruiting Body Formation without the Need for a Host Plant. Mycobiology 48, 263-275.

Kwon H, Yun Y, Kim J, Kim S, Ko H. 2015 - New records of fungi isolated from indoor air of greenhouse used for shiitake cultivation in Korea. Korean Journal of Mycology 43, 58-63.

Maurice S, Arnault G, Nordén J, Botnen SS et al. 2021 - Fungal sporocarps house diverse and host-specific communities of fungicolous fungi. The ISME journal 15, 1445-1457.

Miller MA, Pfeiffer W, Schwartz T. 2010 - Creating the CIPRES Science Gateway for Inference of Large Phylogenetic Trees. Proceedings of the Gateway Computing Environments Workshop (GCE), November 14, 2010, New Orleans, Louisiana 1-8.

Nieto CHD, Granero AM, Zon MA, Fernández H 2018 - Sterigmatocystin: A mycotoxin to be seriously considered. Food and chemical toxicology 118, 460-470.

Pham NDH, Takahashi H, Fukiharu T, Shimizu K et al. 2012 - Phlebopus spongiosus sp. nov. (Boletales, Boletinellaceae) with a sponge-like tissue. Mycotaxon 119, 27-34.

Polacheck I, Nagler A, Okon E, Drakos P et al. 1992 - Aspergillus quadrilineatus, a new causative agent of fungal sinusitis. Journal of Clinical Microbiology 30, 3290-3293.

Rambaut A 2011 - FigTree. Tree figure drawing tool version 1.3.1, Institute of Evolutionary Biology, University of Edinburgh. Available at: http://tree.bio.ed.ac.uk/software/figtree/ (Accessed on August 18, 2021).

Raghoonundon B, Raspé O, Thongklang N, Hyde KD. 2021 - Phlebopus (Boletales, Boletinellaceae), a peculiar bolete genus with widely consumed edible species and potential for economic development in tropical countries. Food Bioscience, 41, 100962.

Ronquist F. Huelsenbeck JP. 2003 - MRBAYES 3: Bayesian Phylogenetic inference under mixed models. Bioinformatics 19, 1572-1574.

Samson RA, Visagie CM, Houbraken J, Hong SB et al. 2014 - Phylogeny, identification and nomenclature of the genus Aspergillus. Studies in mycology 78, 141-173. 
Seyedmousavi S, Lionakis MS, Parta M, Peterson SW, Kwon-Chung KJ. 2018 - Emerging Aspergillus species almost exclusively associated with primary immunodeficiencies. In Open forum infectious diseases, US: Oxford University Press 5, 1-9.

Stamatakis A, Hoover P, Rougemont J. 2008 - A rapid bootstrap algorithm for the RAxML web servers. Systematic Biology 57, 758-771.

Stamatakis A. 2014 - RAxML version 8: a tool for phylogenetic analysis and post-analysis of large phylogenies. Bioinformatics 30, 1312-1313.

Sun JZ, Liu XZ, Jeewon R, Li YL et al. 2019b - Fifteen fungicolous Ascomycetes on edible and medicinal mushrooms in China and Thailand. Asian Journal of Mycology 2, 129-169.

Sun JZ, Liu XZ, McKenzie EH, Jeewon R 2019a - Fungicolous fungi: terminology, diversity, distribution, evolution, and species checklist. Fungal Diversity 95, 337-430.

Wickremasinghe R, Abeywickrama KP, Abeythunga DTU. 1999 - Isolation and identification of fungi from mushroom composts and evaluation their biological activity. Journal of National Science Foundation, Sri Lanka 27, 29-40. 


\section{Supplementary data}

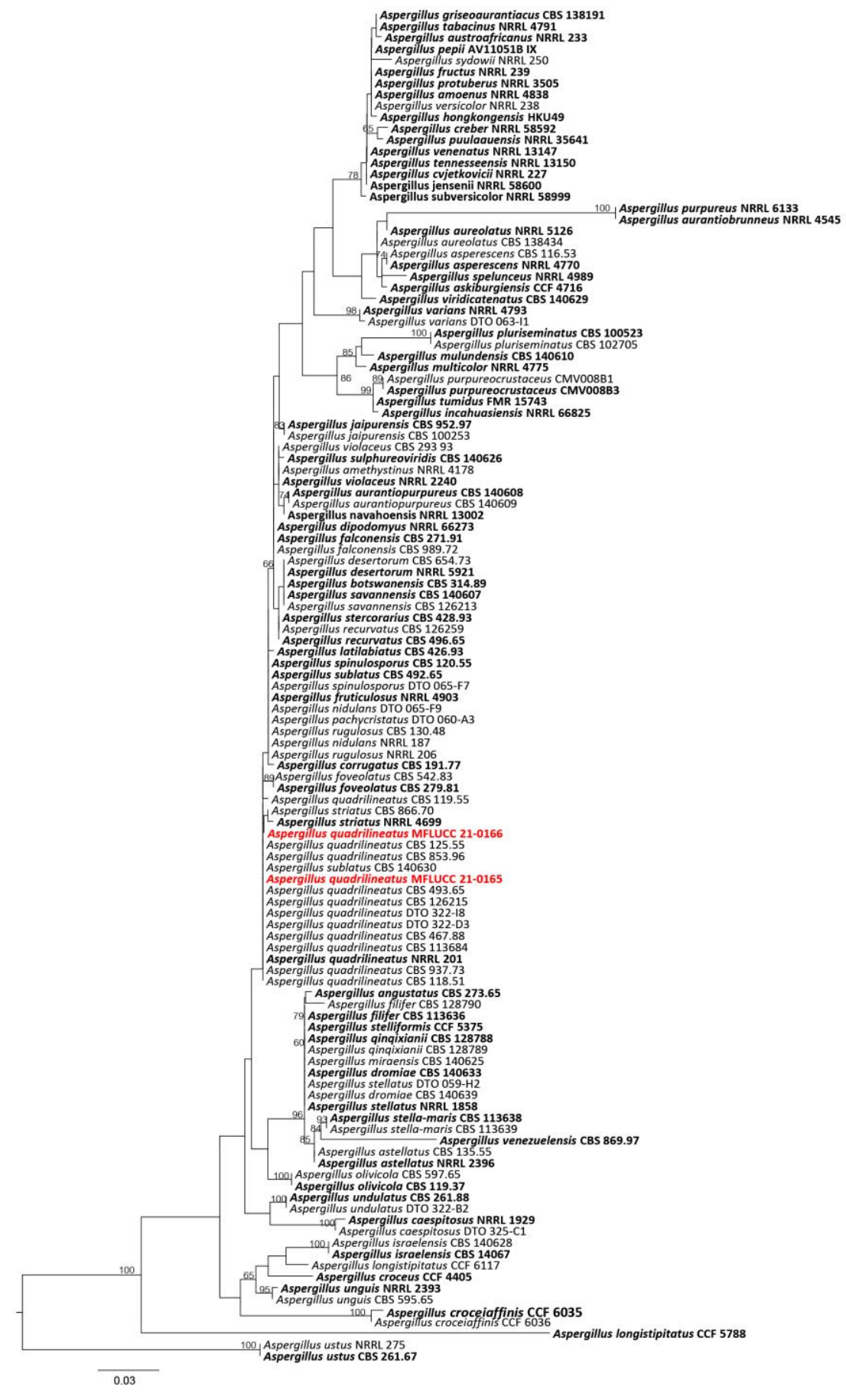

Supplementary Fig. 1 - Phylogram generated from the best scoring of the RAxML tree based on ITS data set. The best RAxML tree with a final likelihood value of -2799.552119 is presented. RAxML analysis yielded 247 distinct alignment patterns and $11.07 \%$ of undetermined characters or gaps. Estimated base frequencies were as follows: $A=0.203407, C=0.291454, G=0.293221, T=$ 0.211918 , with substitution rates $\mathrm{AC}=3.228376, \mathrm{AG}=3.970127, \mathrm{AT}=6.916133, \mathrm{CG}=1.137925$, $\mathrm{CT}=10.928526, \mathrm{GT}=1.000000$. The gamma distribution shape parameter alpha $=0.203658$. Bootstrap support values for maximum likelihood (ML) equal to or greater than $65 \%$; are given above the nodes. Type sequences are in black bold and newly generated sequences are indicated in red bold. 


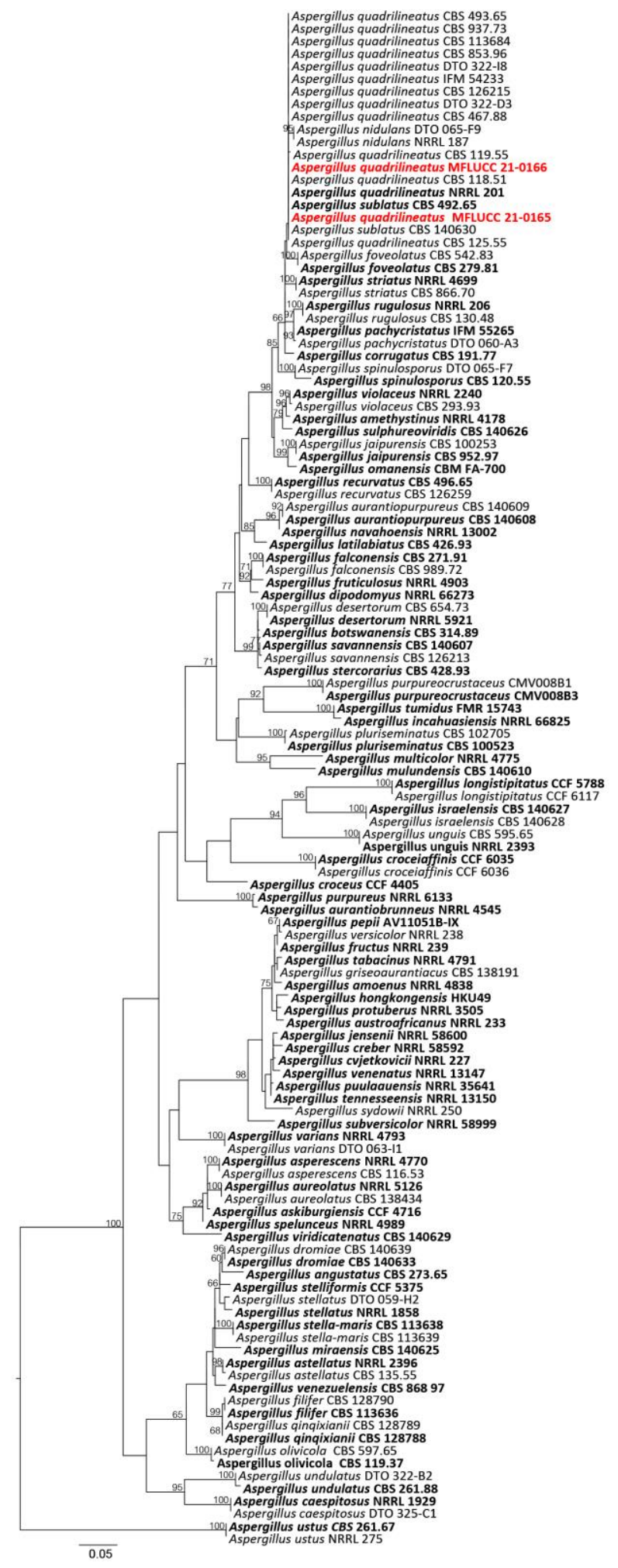

Supplementary Fig. 2 - Phylogram generated from the best scoring of the RAxML tree based on tub2 data set. The best RAxML tree with a final likelihood value of -5209.144157 is presented. RAxML analysis yielded 272 distinct alignment patterns and $12.54 \%$ of undetermined characters or gaps. Estimated base frequencies were as follows: $\mathrm{A}=0.217681, \mathrm{C}=0.300095, \mathrm{G}=0.245345, \mathrm{~T}=$ 0.236879 , with substitution rates $\mathrm{AC}=1.310150, \mathrm{AG}=4.782309, \mathrm{AT}=1.652596, \mathrm{CG}=0.868551$, $\mathrm{CT}=6.245129, \mathrm{GT}=1.000000$. The gamma distribution shape parameter alpha $=0.345865$. Bootstrap support values for maximum likelihood (ML) equal to or greater than 65\%; are given above the nodes. Type sequences are in black bold and newly generated sequences are indicated in red bold. 